\title{
Robust Transmission of H.264/AVC Streams Using Adaptive Group Slicing and Unequal Error Protection
}

\author{
Nikolaos Thomos, ${ }^{1,2}$ Savvas Argyropoulos, ${ }^{1,2}$ Nikolaos V. Boulgouris, ${ }^{3}$ and Michael G. Strintzis ${ }^{1,2}$ \\ ${ }^{1}$ Information Processing Laboratory, Electrical and Computer Engineering Department, Aristotle University of Thessaloniki, \\ Thessaloniki 54124, Greece \\ ${ }^{2}$ Centre for Research and Technology Hellas (CERTH), Informatics and Telematics Institute, Thessaloniki 57001, Greece \\ ${ }^{3}$ Department of Electronic Engineering, Division of Engineering, King's College London, London WC2R 2LS, UK
}

Received 29 July 2005; Revised 12 December 2005; Accepted 18 February 2006

\begin{abstract}
We present a novel scheme for the transmission of H.264/AVC video streams over lossy packet networks. The proposed scheme exploits the error-resilient features of H.264/AVC codec and employs Reed-Solomon codes to protect effectively the streams. A novel technique for adaptive classification of macroblocks into three slice groups is also proposed. The optimal classification of macroblocks and the optimal channel rate allocation are achieved by iterating two interdependent steps. Dynamic programming techniques are used for the channel rate allocation process in order to reduce complexity. Simulations clearly demonstrate the superiority of the proposed method over other recent algorithms for transmission of H.264/AVC streams.
\end{abstract}

Copyright () 2006 Hindawi Publishing Corporation. All rights reserved.

\section{INTRODUCTION}

The demand for multimedia transmission over best effort networks, like the Internet, motivated most recent research on real-time streaming applications. However, due to the explosive growth of the volume of transmitted data and bandwidth variations, networks employing the Internet protocol (IP) exhibit packet erasures. Considering that the network is unaware of the transmitted content, we realize that packet erasures during transmission can cause significant problems in demanding applications such as video streaming. Error-resilient coding schemes like the H.264/AVC standard $[1,2]$ have been proposed to overcome these problems. The H.264/AVC standard supports valuable error-resilient tools to cope with erased packets, while it outperforms previous coding standards (H.263, MPEG-4). Unfortunately, these tools increase the computational complexity, which is undesirable for real-time video applications, and have a negative impact on compression efficiency. Therefore, schemes combining unequal error protection (UEP) algorithms with appropriate selection of error-resilient tools are often shown to be advantageous for transmission of H.264/AVC-coded streams, while maintaining the computational cost at reasonable level.

In a recent work [3], data partitioning of H.264/AVC and high-memory rate compatible punctured convolutional codes (RCPC) [4] were proposed for video transmission over wireless channels. RCPC codes were applied to the network adaptation layer (NAL). Data partitions were unequally protected according to their significance. A similar approach was presented in [5], which also used the data partitioning mode of H.264/AVC. The transmitted data were protected by Reed-Solomon (RS) codes applied at the video coding layer (VCL). Unequal channel rate allocation was performed using Lagrangian optimization techniques. The efficiency of H.264/AVC error-resilient tools was evaluated in [6]. ReedSolomon codes and a feedback channel were considered for robust transmission. Robust transmission of H.263 [7] streams was examined in [8]. A packetization method of slices and an UEP algorithm for joint optimization of macroblock coding parameters and selection of FEC codes were presented.

Partial Reed-Solomon codes (PRS) were used in [9] for reliable transmission of H.264/AVC streams over packet erasure channels. The resulting scheme was able to reduce jerkiness and improve video quality. The concept of key pictures was introduced for H.264/AVC in [10]. The source encoder was appropriately modified to generate packets of unequal importance which are unequally protected. An algorithm which adaptively classifies the data packets of MPEG2 -encoded video streams into two quality of service (QoS) classes was proposed in [11]. Packet classification into priority classes was also studied in [12]. Intraframe interleaving and RS codes were used to improve error resilience. 


\begin{tabular}{|c|c|c|c|c|}
\hline $\begin{array}{c}\text { Encoding } \\
\text { parameters }\end{array}$ & $\mathrm{MB}_{1}$ & $\mathrm{MB}_{2}$ & $\ldots$ & $\mathrm{MB}_{L}$ \\
\hline
\end{tabular}

FIgURE 1: Structure of slices.

The scheme proposed in the present paper is based on macroblock classification and unequal error protection of H.264/AVC streams. Prior to transmission, macroblocks are classified into three slice groups by examining their contribution to video quality. Since the transmission scenarios are over packet networks, facing moderate to high packet loss rates, RS codes are used for channel protection. RS protection is selected for each slice group using a channel rate allocation algorithm based on dynamic programming techniques. To the best of our knowledge, the present method is the first utilizing the explicit mode of the H.264/AVC flexible macroblock ordering (FMO) [13] in conjunction with channel coding techniques. The resulting system is evaluated and is shown to outperform the recently proposed method in [5]. The performance gain is attributed to the more efficient data organization of our scheme, which allows better error concealment without sacrificing coding performance, and to the finer protection of slice groups arising from our unequal error protection strategy.

The paper is arranged as follows. The adaptive macroblock slice grouping employed by the proposed scheme is described in Section 2. Section 3 presents the proposed unequal error protection algorithm. Experimental results are reported in Section 4. Finally, conclusions are drawn in Section 5.

\section{ADAPTIVE MACROBLOCK SLICE GROUPING}

In this section, we present the macroblock classification policy employed by the proposed scheme. Macroblocks are rectangular picture areas and are considered the basic encoding units in H.264/AVC. Although independent encoding of macroblocks is allowed, in general, this approach is not preferable since it would require the transmission of overhead for stating the encoding parameters for each one of the independently encoded macroblocks. To overcome this problem, macroblocks are not coded as single units, but in larger groups of macroblocks, termed slices. Slices are structures of jointly encoded macroblocks which exploit spatial dependencies more effectively by partially sacrificing the error localization capabilities of the decoder. The encoding parameters of macroblocks are declared in a header (Figure 1) which includes the encoding parameters of all macroblocks in a slice. Therefore, slices are self-contained in the sense that they can be independently decoded without utilizing data from other slices of the current frame. Henceforth, each such slice will be assumed to be transmitted in a single transmission unit which will be termed "packet." The terms "packets" and "slices" will be used interchangeably in the analysis below, with "packet" meaning the transmitted stream corresponding to a slice. In this work, we assume that macroblocks are classified in three categories. This is depicted in Figure 3(a). Due to this classification, if a slice is erased, only the macroblocks which are located at slice boundaries can be concealed effectively using neighboring ${ }^{1}$ slices that were received errorlessly at the decoder. Specifically, error-affected frame areas are efficiently concealed using the nonnormative concealment methods of [14].

The limitation of the above conventional slice formation is partially overcome in H.264/AVC, in which error concealment is improved by means of an arrangement which is termed flexible macroblock ordering (FMO). Using FMO, groups of macroblocks, known as slice groups, are formed. Slice groups consist of one or more slices; this enables better error localization. The structure of a slice group is illustrated in Figure 2. Some macroblock classification patterns, like the checkerboard (Figure 3(b)), are available in the H.264/AVC standard. As reported in [15], the FMO mode, in conjunction with advanced error concealment methods applied at the decoder, maintains the visual impact of the losses at a low level even at loss rates up to $10 \%$, which makes it difficult for a trained eye to identify the lossy environment. Apart from predefined patterns, fully flexible macroblock ordering (explicit mode) is also allowed. According to this mode, macroblock classification into slice groups may not remain static throughout the entire video sequence, but it may change dynamically based on the video content.

The provision for dynamic formation of slice groups is exploited by the proposed system. Specifically, slice groups are formed with respect to their relative importance. As a measure of macroblock importance (based on the mean square error, MSE), we use the distortion $D_{\mathrm{MB}}$ defined as

$$
D_{\mathrm{MB}}=\frac{1}{x_{\mathrm{MB}} \cdot y_{\mathrm{MB}}} \cdot \sum_{i=1}^{x_{\mathrm{MB}}} \sum_{j=1}^{y_{\mathrm{MB}}}\left(c_{i, j}-\tilde{c}_{i, j}\right)^{2}
$$

where $x_{\mathrm{MB}}, y_{\mathrm{MB}}$ are macroblock dimensions and $c_{i, j}, \tilde{c}_{i, j}$ are, respectively, the original and the reconstructed coefficients in a macroblock. Alternatively, other metrics like the mean absolute error (MAE) could also be used.

Prior to macroblock classification, the mean value $D_{\text {mean }}$ of the macroblock distortions is computed as

$$
D_{\text {mean }}=\frac{1}{N_{\mathrm{MB}}} \cdot \sum_{i=1}^{N_{\mathrm{MB}}} D_{\mathrm{MB}_{i}}
$$

where $N_{\mathrm{MB}}$ is the total number of macroblocks in a frame and $D_{\mathrm{MB}_{i}}$ is the distortion associated with the $i$ th macroblock. Subsequently, the relative distortion of each macroblock is compared with $D_{\text {mean }}$. The macroblocks are labelled with respect to their importance as "high," "medium," and "low" as in [12]. The classification of the macroblocks into the above categories takes place using two thresholds, $T_{l}$ and $T_{h}$,

\footnotetext{
1 The term neighboring refers to both the spatial and the temporal domains. Thus, slices from the current and the previous frames are used for error concealment.
} 


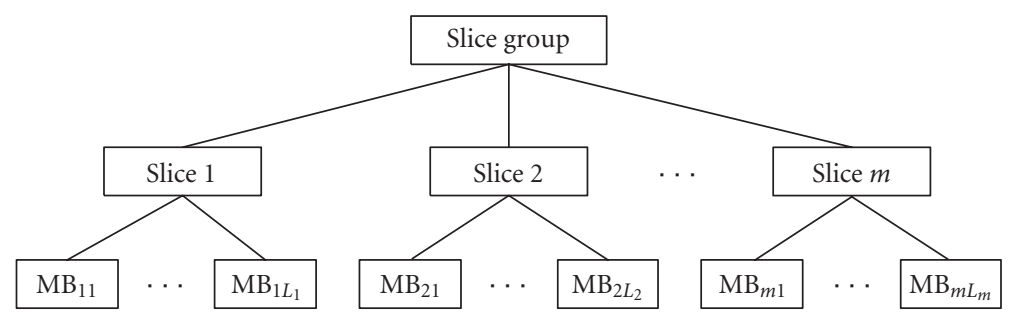

FIGURE 2: Slice group formation.

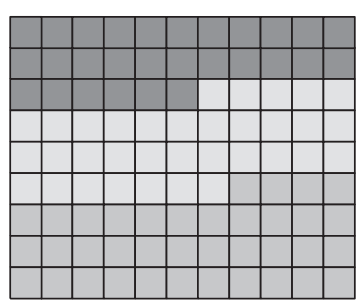

(a)

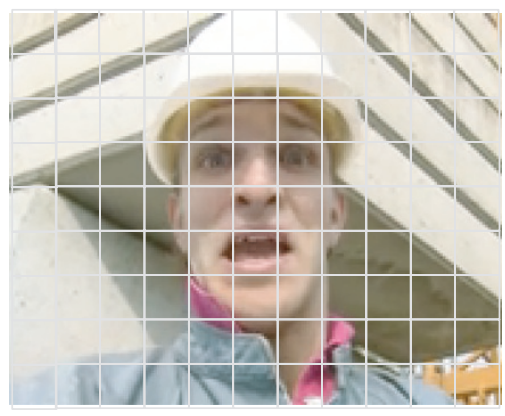

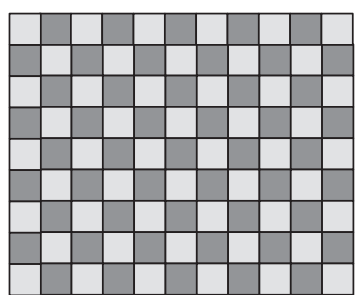

(b)

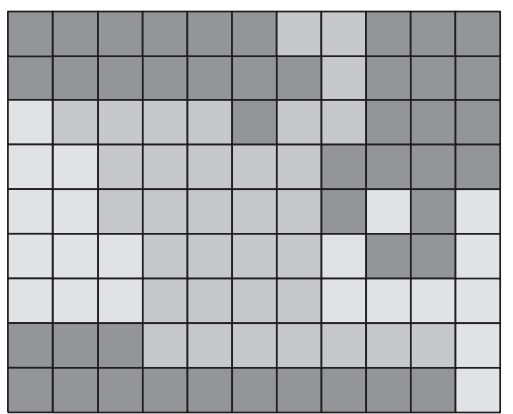

Slice group 1

Slice group 2

Slice group 3

(c)

(d)

FIGURe 3: Macroblock classification (a) without FMO, (b) employing FMO (checkerboard), (c) original frame of Foreman, (d) classification map following fully FMO mode.

according to the following rules:

(i) if $D_{\mathrm{MB}}<T_{l} \cdot D_{\text {mean }}$, the examined macroblock is classified to the "low" importance slice group,

(ii) if $T_{l} \cdot D_{\text {mean }} \leq D_{\mathrm{MB}}<T_{h} \cdot D_{\text {mean }}$, the examined macroblock is classified to the "medium" importance slice group,

(iii) if $D_{\mathrm{MB}} \geq T_{h} \cdot D_{\text {mean }}$, the examined macroblock is classified to the "high" importance slice group.

The distortion $D_{\mathrm{MB}}$ initially used is determined assuming the frame as a single slice group. After the classifica- tion of macroblocks into three slice groups, the compression efficiency will degrade and thus, more bits will be needed for the encoding of each macroblock than those initially estimated. This is taken into account by the rate-control algorithm at the encoder. In Figures 3(c) and 3(d), a frame of the Foreman sequence and its macroblock allocation map (MBAmap) for three classes, according to the above rules, are presented. The area regarded as being of high-importance mainly corresponds to intense motion or high texture regions. For example, in Figure 3(c) the "high" importance slice group coincide with foreman's head which is the main 


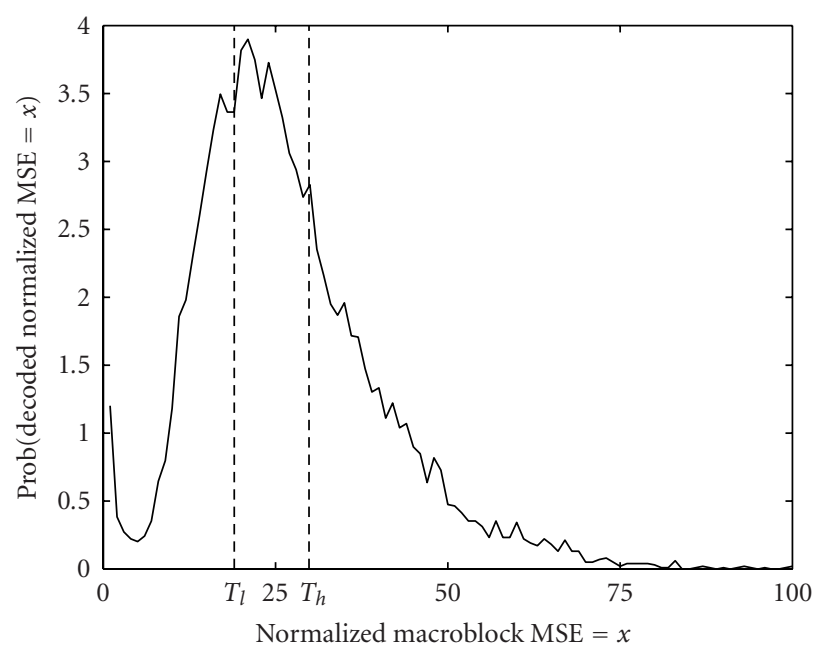

FIGURE 4: Histogram function of macroblocks distortion and their respective classification thresholds.

moving object in the scene, whereas the background and the body are signed as medium and low importance slice groups.

The classification of macroblocks into three categories, and not more, is reasonable, since in this way macroblocks of approximately equal importance are grouped together. Classification into more categories would not be preferable because it would lead to the generation of rather small-length packets. This is undesirable because of the increased associated packet overhead (RTP/UDP/IP overhead) containing the transmission parameters.

The determination of the thresholds $T_{l}$ and $T_{h}$, which are used for the classification of macroblocks into three slice groups, will be described in Section 3. The average values of $T_{l}$ and $T_{h}$ are 0.7 and 1.1 , respectively. It is worth noting that these threshold values are used only for the initial classification of the optimization algorithm of Section 3. These are subsequently refined during the optimization procedure. The normalized histogram function of macroblocks' distortions and the respective thresholds are illustrated in Figure 4. Following the above classification rules, slice groups are formed.

Since the transmission scenario is over packet erasure networks, channel codes should be used for the efficient protection of the H.264/AVC streams. To this end, we developed an algorithm for the efficient channel rate allocation. This is presented in the ensuing section.

\section{CHANNEL RATE ALLOCATION}

In the preceding analysis for an optimal classification, it was assumed that the distortion between the original and reconstructed coefficients is known. In practice, however, the actual distortion depends on the reconstructed coefficients after channel decoding. This means that the processes of slice grouping and channel allocation are actually interdependent. For this reason, the formation of slice groups and their unequal error protection are optimized in our system by iterating two interdependent steps.
During the channel rate allocation process, slices are transferred from one slice group to another leading to new slice group formations. The channel rate allocation algorithm classifies optimally the macroblocks into slice groups and determines their optimal channel protection. As it can be seen, the choice of the classification thresholds is an important issue. When the thresholds are close to the optimal values, the channel rate allocation procedure is made more efficient and the computational cost is significantly reduced. The thresholds used for classification at the I-frame are initially determined by experimentation and guarantee satisfactory image quality and error resiliency at the receiver. In the sequel, the thresholds are refined following an iterative technique which is described in detail below. Specifically, the resulting macroblock classification is used for the refinement of the classification thresholds. The determined thresholds are used for the initial macroblock classification in the next frame. Similarly, thresholds are determined for the remaining frames. From the above analysis, it is obvious that the FMO generates slices which can be used in conjunction with unequal error protection (UEP) schemes.

\subsection{Problem formulation}

Using the FMO, it is possible to form slice groups of unequal importance. In our approach, the unequally-important slice groups consist of equally sized slices (packets), that is, the size of the slices in each slice group is the same (in bytes) but the importance of the resulting slice groups is different. Therefore, UEP should be applied for their efficient protection. Reed-Solomon (RS) codes were chosen for use with our system due to their excellent error recovery properties for transmission over packet erasure networks. Since, different frames have, in general, different classification maps, channel rate allocation is performed at the frame level. The proposed algorithm takes into account the importance of each slice group and allocates more RS packets (RS slices) to slice groups car- 


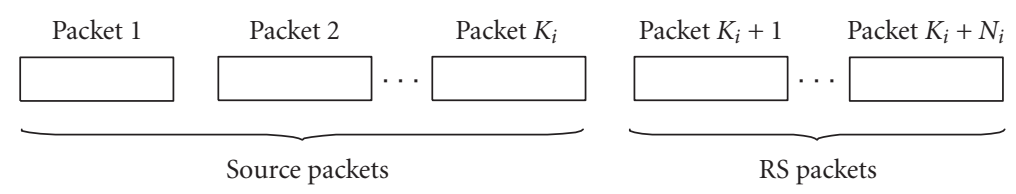

FIGURE 5: Packet formation of a slice group.

rying important information and less to the rest. The problem is solved optimally using dynamic programming techniques under two constraints which are presented in the following. The packet formation of a slice group after RS encoding is illustrated in Figure 5.

The distortion $D_{f}$ of each frame is expressed as the sum of the individual slice group distortions $D_{f, i}$. Therefore,

$$
D_{f}=\sum_{i=1}^{s} D_{f, i}
$$

where $s$ is the number of slice groups.

The optimization objective is to find

(i) the optimal classification of macroblocks into slice groups,

(ii) the optimal RS channel protection of slice groups.

The optimization algorithm intents to minimize the average expected distortion $\bar{D}$ subject to two constraints. The first constraint is imposed by the rate control algorithm of the H.264/AVC. Hence,

$$
\sum_{i=1}^{s} K_{i}=K_{f}
$$

where $K_{i}$ is the number of source packets classified into the $i$ th slice group of a frame, and $K_{f}$ is the total number of source packets for the frame.

A channel rate constraint is required to set an upper limit to the RS protection which can be used for the protection of a frame. This reduces significantly the possible channel rate allocations and facilitates the allocation procedure. Thus, it is

$$
\sum_{i=1}^{s} N_{i} \leq N_{f}
$$

where $N_{i}$ is the number of RS packets allocated to the $i$ th slice group and $N_{f}$ is the total number of RS packets allowed for the protection of the frame.

The channel rate constraint is necessary to avoid overprotection of the first frames. Specifically, without the channel rate constraint, the first frames in the sequence would allocate the maximum allowable RS protection. Therefore, the remaining frames would have less available rate and, consequently, drift would occur. The maximum number $N_{f}$ of RS packets (per frame) which can be used for the channel protection of a frame was found by experimentation. $N_{f}$ is expressed as a fraction of the available source packets for each frame. In order to determine $N_{f}$ and, thus, the optimal channel rate $r_{c}$ of a sequence, the average expected distortion is computed for a large set of channel rates. The $r_{c}$ is given by

$$
r_{c}=\frac{\sum_{i=1}^{N_{\mathrm{seq}}} N_{f, i} \cdot p_{l}}{r_{T}}
$$

where $N_{\text {seq }}$ is the number of frames in a sequence, $N_{f, i}$ the number of RS packets in frame $i, p_{l}$ the packet length, and $r_{T}$ the overall transmission bit rate.

From the computed channel rates $r_{c}$, the one achieving the lowest distortion is considered as optimal. Therefore, the available bit rate for source encoding of the sequence is $r_{s}=$ $\left(1-r_{c}\right) \cdot r_{T}$.

The average expected distortion when all packets are clustered to the same slice group is defined as

$$
\bar{D}=\sum_{i=1}^{N} D_{f} \cdot P(i)+\sum_{i=N+1}^{N+K-1} D_{f, i, 1} \cdot P(i)+D_{f, P C} \cdot P(N+K),
$$

where $K, N$ are the number of source and channel packets, respectively, and $D_{f}$ is the distortion when the number of erased packets do not exceed the allocated RS protection. $D_{f, i, 1}$ (1 stands for the slice group index) is the distortion when concealment is invoked to mitigate the effect of the lost packets. $D_{f, P C}$ denotes the distortion in case all packets of the current frame are lost and frame replication follows for error concealment. In the preceding analysis, the channel rate allocation algorithm assumes that all previous frames have been received intact. Thus, no distortion is introduced due to error propagation. Although, this assumption rarely holds, in general, the resulting allocation is barely affected. Finally, $P(i)$ is the probability that $i$, out of $N+K$, packets are erased. It is found to be equal to

$$
P(i)=\left(\begin{array}{c}
N+K \\
i
\end{array}\right) \cdot p^{i} \cdot(1-p)^{N+K-i},
$$

where $p$ is the packet erasure probability associated with the channel.

We have already defined the average expected distortion when each frame is transmitted as a single slice group. Trivially, it can be proved that the expected distortion for $s$ classes is given by

$$
\begin{aligned}
\bar{D}=\sum_{l=1}^{s}\left\{\sum_{i=1}^{N_{l}} D_{f, l} \cdot P_{l}(i)+\sum_{i=N_{l}+1}^{N_{l}+K_{l}-1} D_{f, i, l}\right. \\
\left.\cdot P_{l}(i)+D_{f, P C, l} \cdot P_{l}\left(N_{l}+K_{l}\right)\right\},
\end{aligned}
$$




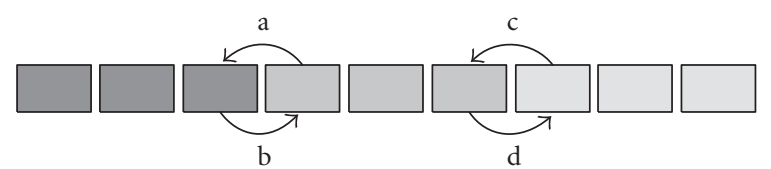

(a)

a

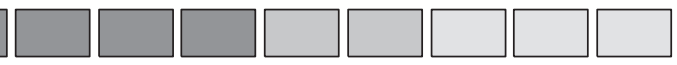

b

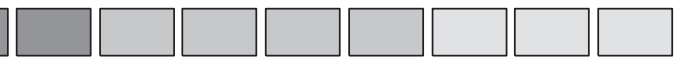

C

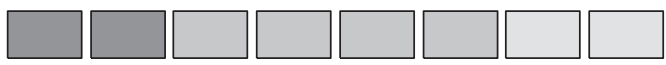

$\mathrm{d}$

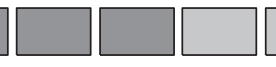

(b)

Figure 6: Allowable packet exchanges in case of three slice groups.

where $K_{l}$ and $N_{l}$ are the number of source and RS packets of the $l$ th slice group. $P_{l}(i)$ is the packet error probability of $l$ th slice group. It is defined similar to (10) as

$$
P_{l}(i)=\left(\begin{array}{c}
N_{l}+K_{l} \\
i
\end{array}\right) \cdot p^{i} \cdot(1-p)^{N_{l}+K_{l}-i} .
$$

The distortion $D_{f, P C, l}$ in the last term of (9) expresses the distortion when all packets of the $l$ th slice group are erased and concealed by slice group replication. Finally, $D_{f, i, l}$ represents the distortion introduced when the current frame slice group is concealed by slices received intact and $D_{f, l}$ the distortion when the RS protection is sufficient to recover all erased packets. It should be noted that the distortion terms do not consider error propagation. This does not affect seriously the estimated distortion since macroblocks updates usually cope effectively with drift phenomenon.

\subsection{Reed-Solomon rate allocation}

In this section, we present a solution to the optimization problem that was previously formulated. The optimization objective is actually two fold. Specifically, it includes the determination of both the number of slices that are classified into each slice group and their respective RS protection. In general, reaching an optimal solution of the above joint optimization problem is a difficult task. In this work, we propose a two-step optimization procedure, which iteratively determines the packet classification and the RS protection. Although, this approach to the solution of the optimization problem does not guarantee global optimization, in practice it yields very satisfactory results. The optimization procedure is summarized as follows.

(1) Determine the RS protection for each frame.

(2) Determine the thresholds $T_{h}$ and $T_{l}$.

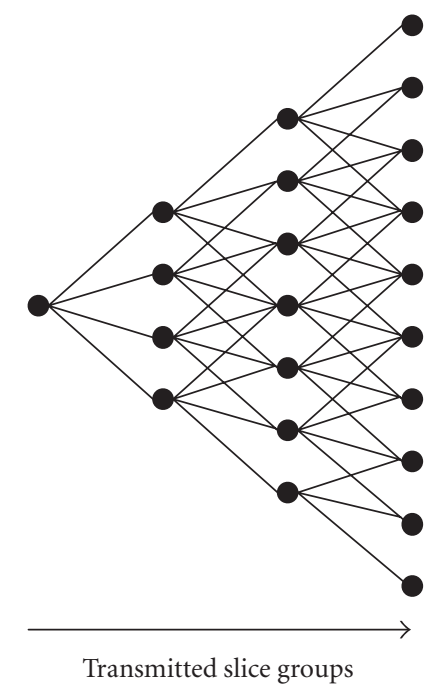

FIgURE 7: Trellis diagram for RS allocation.

(3) Classify all macroblocks into slice groups according to $T_{h}$ and $T_{l}$.

(4) Find the optimal RS protection for the above classification.

(5) Calculate the expected distortion of allowable neighboring macroblock classifications with the restriction that a single packet can be exchanged between successive classes.

(6) Compare the expected distortion of the ancestor classification with the lowest average distortion of all descendant classifications of step (3). If a classification with lower expected distortion is reached, it is considered as optimal and steps (2) to (6) are repeated, otherwise the algorithm is terminated. When the same packet is exchanged between two slice groups in two successive iterations, the algorithm is again terminated.

If three slice groups are assumed, the possible packet exchanges are illustrated in Figure 6. It is worth noting that the actual search space is limited, since only four new packet formations are possible. If a slice group does not contain any packet, the possible formations are even fewer.

Our objective is to optimize the RS allocation by minimizing the expected distortion given by (9). Although this optimization can be performed by exhaustive search among all possible channel rate allocations, this approach is not preferable since the computational cost would be prohibitive for real-time applications. However, the computational cost can be significantly reduced using the dynamic programming algorithm in $[16,17]$. The trellis diagram corresponding to the minimization of (9), subject to a rate constraint, is shown in Figure 7. Each branch in the trellis corresponds to the application of a specific RS code to a slice group. The algorithm first determines the RS protection of the more important slice groups and then the respective protection of the 
less important slice groups. The nodes in the trellis represent the intermediate stages where decisions are made about the best RS allocation up to the sth slice group protection. Paths merging in a single node correspond to allocations that yield not only the equal source rates but also equal transmission rates. Among the paths converging to a node, the path attaining the lower expected distortion is retained (survivor) while the rest are pruned. In the final stage, among the survivor paths, the one with the lowest overall expected distortion corresponds to the optimal RS allocation. The number of states in the trellis depends on the allowable RS protection levels.

\section{EXPERIMENTAL RESULTS}

The proposed scheme for transmission of H.264/AVC streams over IP/UDP/RTP was evaluated using the two standard QCIF sequences Foreman and Carphone, coded at 10 frame/s (fps), and the CIF sequence Paris, coded at $30 \mathrm{fps}$. Group of pictures (GOPs) of IPPP ... structure consisting of 100 and 300 frames were considered for the QCIF and CIF sequences, respectively. The NS-2 event simulator [18], employing a uniform bit error model, was used for channel simulations. The NS-2 was selected to simulate more realistically $^{2}$ the examined wireline transmission scenaria. It should be noted that, with minor modifications, the proposed method could also be used for wireless video transmission.

The video sequences were encoded using JM 8.3 [19] of the H.264/AVC standard [1]. The first frame in the sequence was intracoded and the following frames were intercoded. Temporal redundancy was removed using up to $1 / 4$ pixel accuracy motion compensation. Multiple reference picture selection [20] was allowed for improved coding efficiency and error resiliency. The reference frame buffer was set to the maximum value 5 . The universal variable length coding (UVLC) [1] was selected as the entropy coder. For the estimation of the end-to-end distortion, 30 independent channel-decoder pairs were used in the encoder, as suggested in [21], and nonnormative advanced error concealment methods were applied [14]. The same error concealment techniques were also applied at the decoder side.

The JM 8.3 was modified to support fully flexible macroblocks allocation map (MBAmap) for each frame. The picture parameter set (PPS) packets used by JM 8.3, which contain the classification maps, are protected using strong channel codes. Specifically, the $(3,1)$ RS codes were used since they are able to correct all possible error patterns occurring in the considered channel conditions. The use of these RS codes is affordable because the PPS packet size is small in comparison to the average frame size. In particular, PPS packets sized 30 and 120 bytes on average for QCIF and CIF

\footnotetext{
${ }^{2}$ NS-2 considers several parameters like round trip time, delay, jitter, and advanced features (e.g., drops due to congestion and bottleneck effects in concurrent flows). Although these features are not considered in our experiments, we use NS-2 for channel modelling since it is a well-known testbed and the results can be easily replicated from other researchers.
}

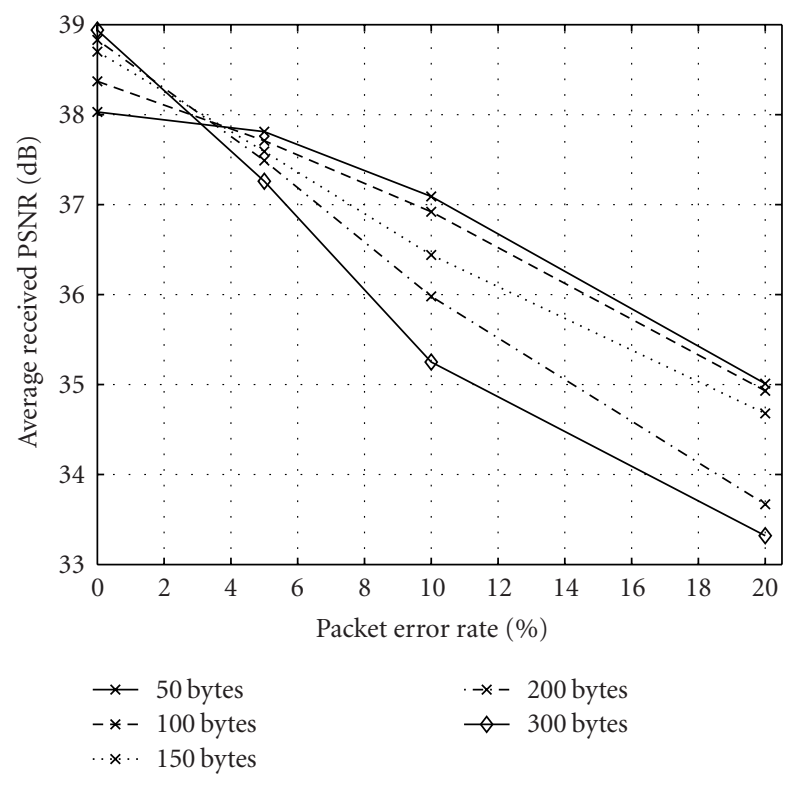

FIgURE 8: Average received mean PSNR for transmission of the Foreman sequence coded at $128 \mathrm{kbps}$ over channels facing packet error rates in the range $[0,20]$ for various packet sizes.

sequences, respectively, while the average frame size was between 800 and 1500 bytes for QCIF sequences and between 3000 and 6000 bytes for CIF sequences. The bit rate allocated to PPS packet protection was in the range of $5-10 \%$ of the overall transmission rate. The chosen channel coding strategy for PPS packets is needed in order to ensure that highquality video sequences will be decodable even in the case of high packet error rates. Due to the strong protection that is applied to the PPS packets, in the sequel we assume that PPS packets are always available without errors at the decoder.

The packet sizes were 50 and 200 bytes for the QCIF and CIF sequences, respectively. The use of relatively small packet sizes endowed our scheme with the ability to achieve better error localization and prevent drift. If longer packets were used, wider frame areas would be affected in case of erasures. In such cases, errors would not be concealed effectively and the decoding process would be inefficient. The main drawback of utilizing small packets is, as expected, the less efficient compression due to the poor prediction and the increased packet overhead. This is shown in Figure 8 where it is seen that small packets guarantee the decoding of video sequences of satisfactory quality, whereas schemes with larger packets benefit in error-free cases. Considering the above, our choices of packet sizes achieve a good tradeoff between robustness and compression efficiency.

The employment of small packets could result in increased bandwidth requirements for packet headers transmission. In order to avoid this, the robust header compression (RoHC) [22] was used, which reduces the IP/UDP/RTP header from 40 bytes to approximately 3 bytes. Thus, the resulting packet overhead is about $1.5 \%$ and $6 \%$ of the overall 


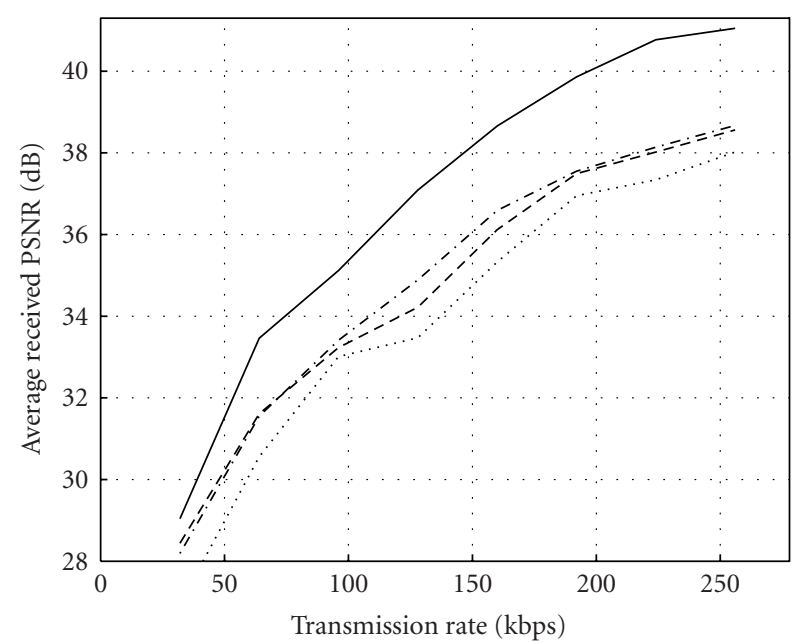

— Proposed method, three slice groups

-.- $[5]$

Proposed method, single slice group

- - - Proposed method, checkerboard

(a)

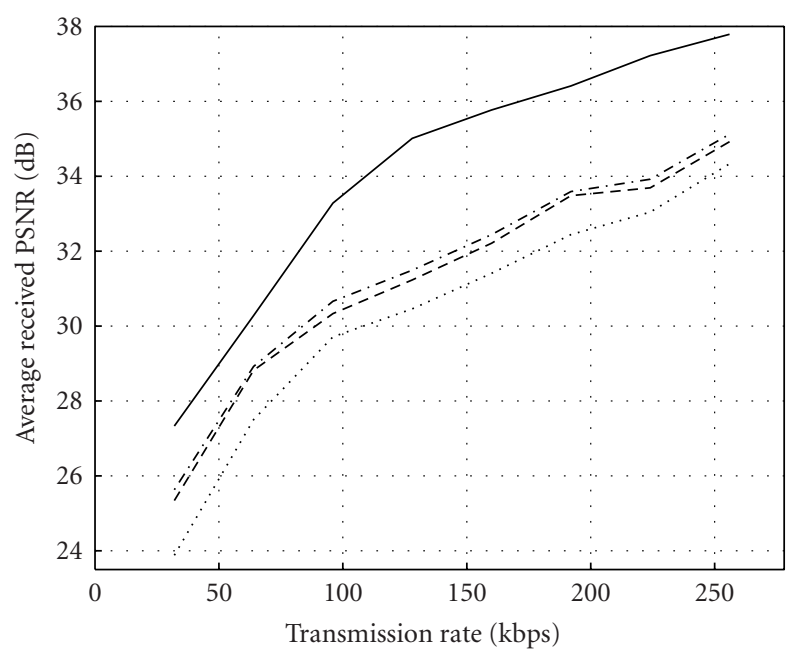

— Proposed method, three slice groups

-. - $[5]$

..... Proposed method, single slice group

- - - Proposed method, checkerboard

(b)

FIGURE 9: Comparison of the proposed methods with the method in [5] for the transmission of the QCIF sequence Foreman. Reconstruction quality in terms of mean PSNR is reported. Results for packet error rate equal (a) $10 \%$, (b) $20 \%$.

transmission rate for CIF and QCIF sequences, respectively. This cost is reasonable considering that small packets improve drastically the error concealment and localization capabilities of the system. The main disadvantage of RoHC is the increased processing delay at routers, which leads to endto-end delays. However, as shown in several other techniques (e.g., in [23-27]) it is possible to use RoHC for real-time communication over multihop networks.

Adaptive slice grouping was employed by the proposed system. Specifically, as presented in Section 2, the slices were classified into three slice groups. The MSE was considered as the classification metric. Since, the slice groups are of unequal importance, different sets of RS code rates were used for their protection. Therefore, the slice groups labelled as "low" and "medium" are protected less, while stronger RS codes were used for the class of "high" importance.

Three variants of the proposed scheme were considered for comparison purposes:

(i) the full scheme, which classifies macroblocks into three slice groups according to the rules presented in Section 2,

(ii) a scheme which divides the image into two slice groups according to the checkerboard pattern,

(iii) a simplified scheme which treats each frame as a single slice group.

The RS protection for the above schemes was determined using the UEP algorithm of Section 3. Prior to channel rate allocation the optimal channel rate $r_{c}(6)$ is found. Then the algorithm follows the optimization process presented in Section 3.2, which iteratively refines the estimated RS protec- tion until a close to optimal protection is reached. From the examined RS allocations, the strongest employed RS code is the one which allocates all RS packets to the most important slice group. In particular, if $K_{i}$ is the number of source packets of the $i$ th slice group, then the examined RS codes are part of the $\left(K_{i}+\xi, K_{i}\right)$ family, where $\xi \in\left[0, N_{f}\right] .^{3}$

The peak-signal-to-noise ratio (PSNR) was used as a measure of the reconstruction quality. As in almost all related literature, in the present work we report results in terms of mean PSNR. All reported results are averages over 100 simulations. The proposed schemes are compared with an implementation of the method in [5] which uses two data partitions and employs slices of fixed number of macroblocks. The optimization of [5] was applied at the NAL level. The method in [5] was selected for comparison purposes since it is a joint source/channel coding scheme which is in the spirit of our method. The transmission schemes were evaluated for a variety of channel conditions. In Figures 9(a), 10(a), and 11(a), results for transmission over packet networks with $10 \%$ packet losses are presented for the Foreman, Carphone, and Paris video sequences. Optimization was performed assuming 10\% packet error rate. From Figures 9(a), 10(a), and 11(a), it can be easily seen that the three slice group variant of the proposed method decodes higher-quality videos more frequently than the rest of the methods. The performance gap between our best-performing scheme and the method in [5] is significant and grows wider as the transmission bit rate

\footnotetext{
${ }^{3}$ Typical values for $K_{i}$ and $N_{f}$ range from 3 to 10 and from 0 to 10 , respectively.
} 


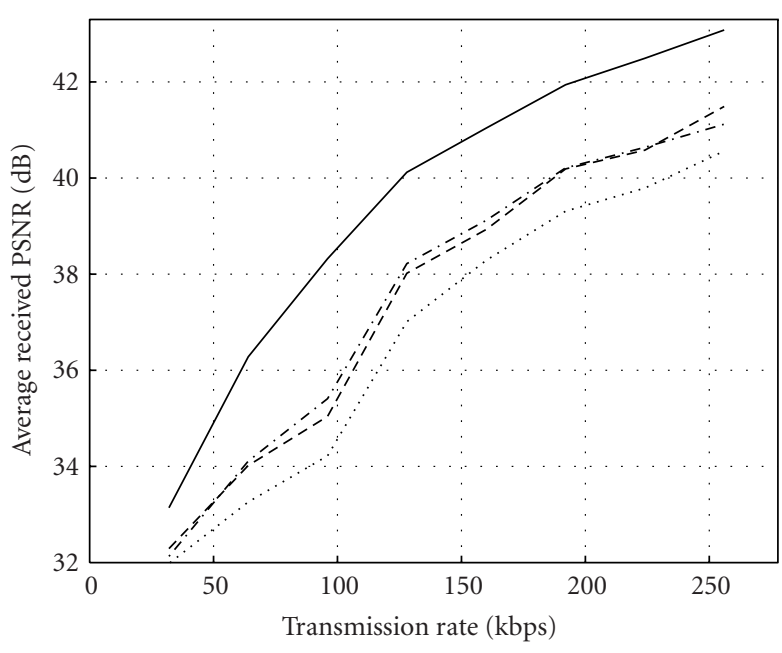

_ Proposed method, three slice groups

-.. [5]

..... Proposed method, single slice group

- - - Proposed method, checkerboard

(a)

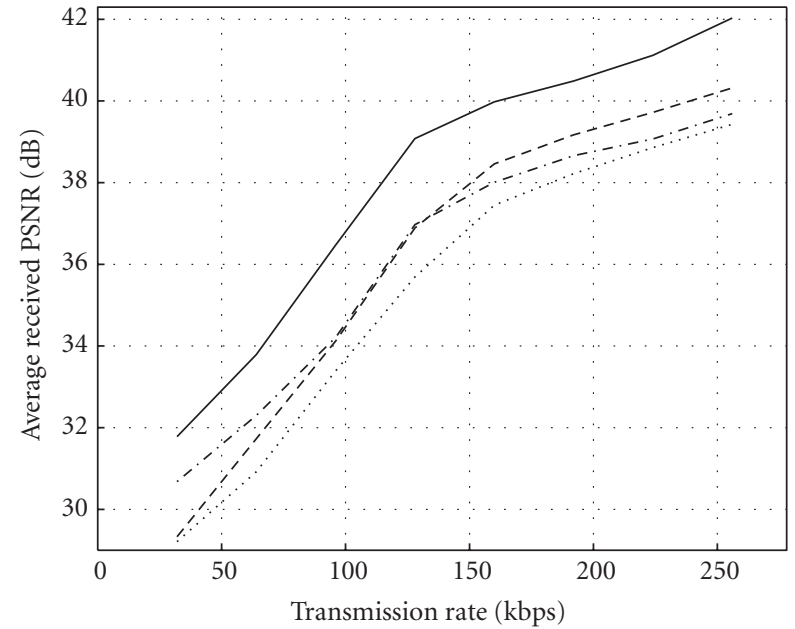

_ Proposed method, three slice groups

-..- $[5]$

..... Proposed method, single slice group

- - Proposed method, checkerboard

(b)

FIGURE 10: Comparison of the proposed methods with the method in [5] for the transmission of the QCIF sequence Carphone. Reconstruction quality in terms of mean PSNR is reported. Results for packet error rate equal (a) $10 \%$, (b) $20 \%$.

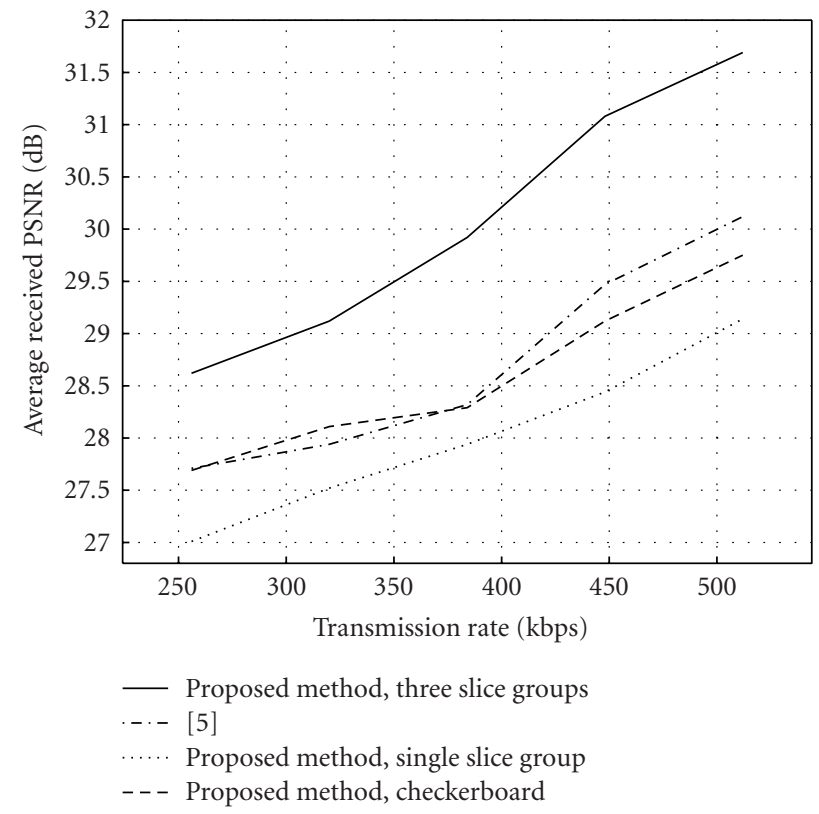

(a)

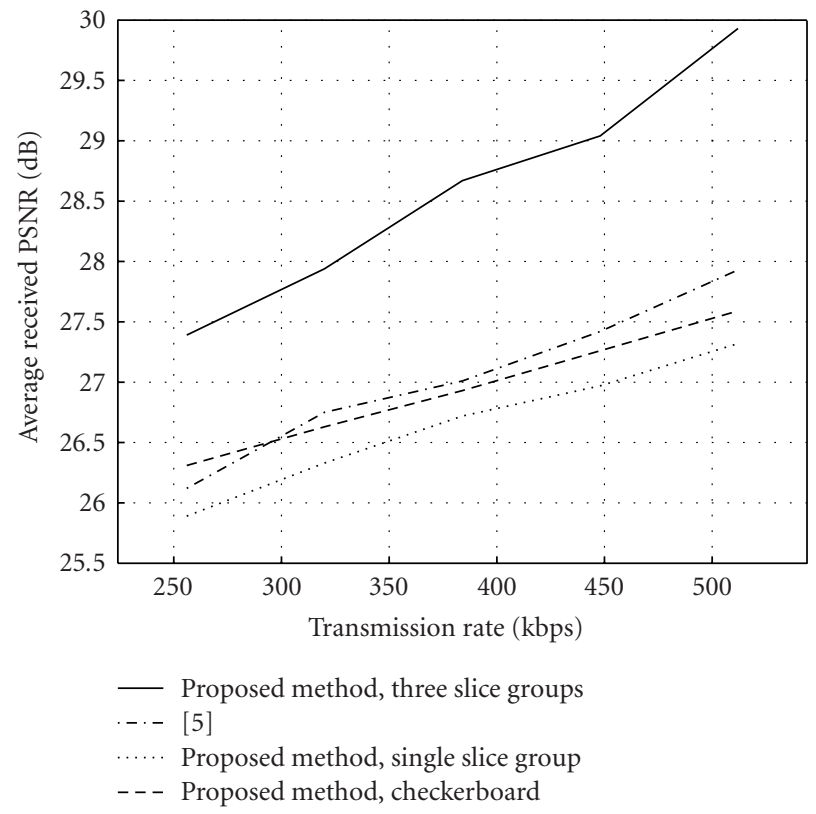

(b)

FIGURE 11: Comparison of the proposed methods with the method in [5] for the transmission of the CIF sequence Paris. Reconstruction quality in terms of mean PSNR is reported. Results for packet error rate equal (a) $10 \%$, (b) $20 \%$.

increases. The performance gains achieved using the proposed scheme is due to the adaptive slice grouping which enables better error localization as well as the efficient error protection. From Figures 9, 10, and 11 it is obvious that our three slice group approach performs significantly better than other variants of our scheme (i.e., single-sliced scheme). The unequal error protection algorithm also boosts the performance of the proposed scheme, since the unequal protection 


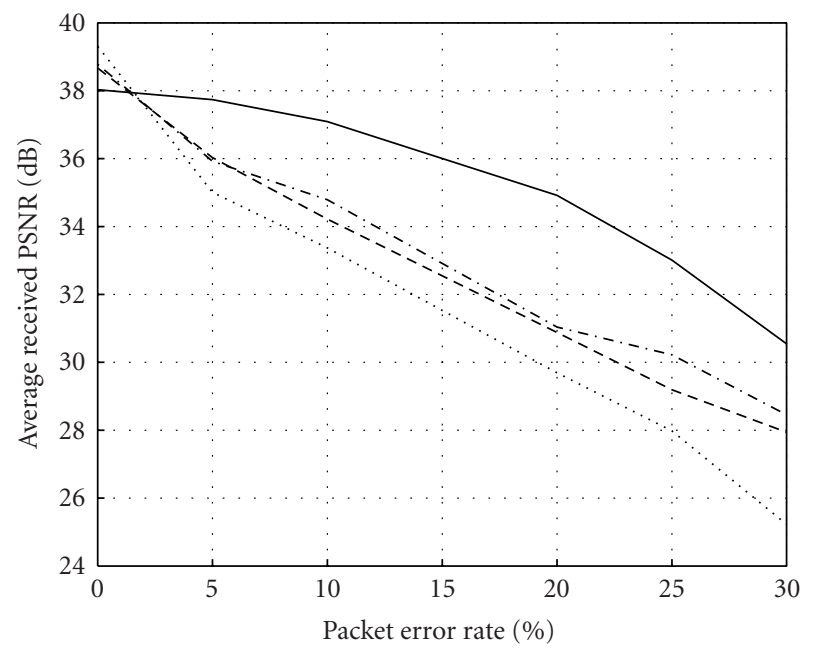

_ Proposed method, three slice groups

$\cdot-\cdot-[5]$

..... Proposed method, single slice group

- - - Proposed method, checkerboard

FIGURE 12: PSNR comparison for the transmission of the QCIF sequence Foreman at $128 \mathrm{kbps}$ as a function of the packet error rate. The scheme was optimized for $10 \%$ packet error rate and tested for various packet error rates.

of slice groups enables the application of less powerful RS codes, and thus, saves rate which can be used for the transmission of source rate. Considering the above, the performance gain should not be attributed solely to the adaptive group slicing itself or the UEP algorithm, but rather to their synergistic cooperation.

Transmission of video over more unreliable channels was also considered. The schemes were optimized for $20 \%$ packet error rate and transmitted over packet erasure networks which encounter the considered channel conditions. For the Foreman, Carphone, and Paris sequences the results are presented in Figures 9(b), 10(b), and 11(b), respectively. The results clearly and consistently demonstrate the superiority of the proposed scheme with multiple slice groups and verify the conclusions reached for less noisy channels. As previously, the performance gain stems from both the slice group classification and the optimal channel rate allocation algorithm.

The proposed scheme was also evaluated for transmission in channel mismatch conditions. In Figure 12, results are presented for Foreman QCIF sequence coded at $128 \mathrm{kbps}$ for the case where the schemes are optimized for packet error rate equal to $10 \%$ and transmitted over channels which exhibit various packet error rates. The results show that the proposed full scheme is superior to the method in [5] and the other variants of the full scheme. When the transmission is error free, the proposed full scheme has lower performance due to the application of stronger RS codes and the inferior compression efficiency when FMO is used. The gain achieved by the full scheme over the other methods becomes more impressive when the channel conditions deteriorate. Specifi-

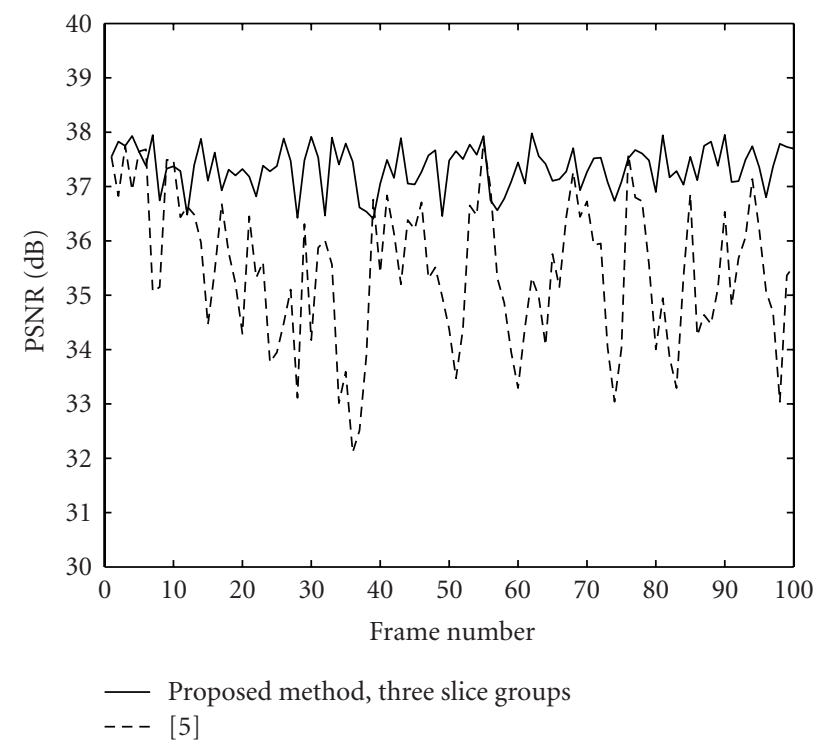

FIGURE 13: PSNR comparison of the proposed full scheme with the method in [5] for the transmission of the QCIF sequence Foreman coded at $128 \mathrm{kbps}$ over packet erasure channel with $10 \%$ packet losses.

cally, for the most of the considered transmission scenarios, the performance gap is roughly $2 \mathrm{~dB}$. It is worth noting that our three slice group method provides graceful degradation in image quality when the channel becomes noisier, whereas the other methods collapse. This is due to the exploitation of adaptive slice grouping which improves the performance of error concealment methods and the channel rate allocation algorithm of Section 3.

For the sake of the comparison, in Figure 13 the full scheme is compared, in terms of PSNR, with the method in [5] for transmission of Foreman over channel with 10\% packet losses. As it can be seen, the proposed scheme is, in general, more robust to packet losses. Moreover, the reconstruction quality degrades more gracefully. On the contrary, the method in [5] exhibits unpleasant fluctuations in image quality.

In Figure 14, we present a visual comparison of the decoded sequences by the proposed methods. From Figure 14, we can see that the three slice group variant of the proposed method outperforms the other variants. It should also be noticed that the proposed method does not induce annoying artifacts.

\section{CONCLUSIONS}

A novel method was proposed for the transmission of H.264/AVC-coded sequences over packet erasure channels. The proposed scheme exploits the error resilient features of H.264/AVC codec and employs Reed-Solomon codes to protect effectively the resulting streams. A novel macroblock classification scheme into three slice groups was used for 


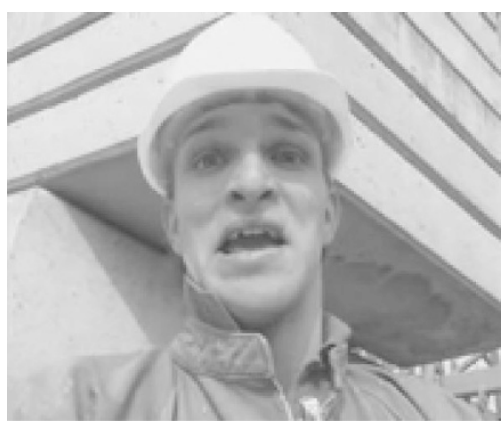

(a)

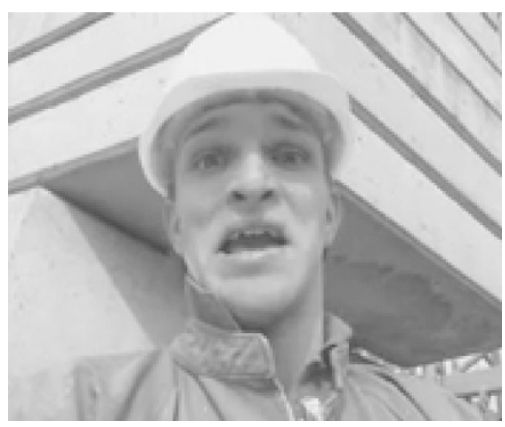

(c)

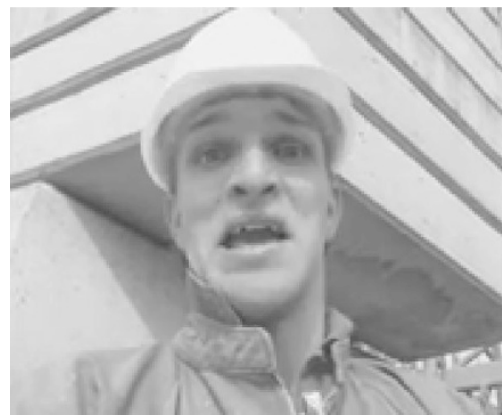

(e)

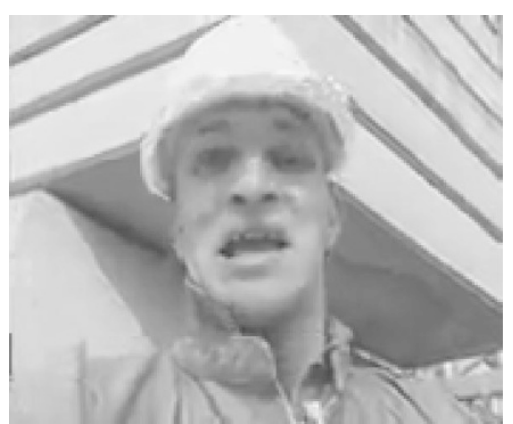

(b)

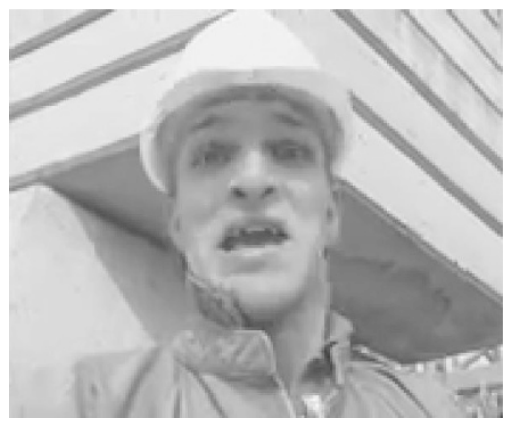

(d)

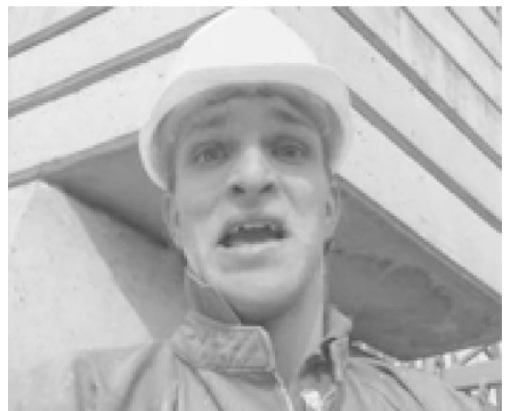

(f)

FIgURE 14: Visual comparison of the proposed methods using the frame 68 of the Foreman sequence coded at $96 \mathrm{kbps}$. Comparison of visual artifacts induced due to transmission over packet networks encountering $10 \%$ packet error rate. Error-free transmission of the (a) single slice group variant of the proposed scheme $(37.32 \mathrm{~dB})$, (c) two slice groups (checkerboard) variant of the proposed scheme (36.58 $\mathrm{dB}$ ), (e) three slice groups variant of the proposed scheme $(36.06 \mathrm{~dB})$. Frames harmed by noise when sequences are encoded using the (b) single slice group variant of the proposed scheme $(32.86 \mathrm{~dB})$, (d) two slice groups (checkerboard) variant of the proposed scheme (33.47 dB), (f) three slice groups variant of the proposed scheme $(34.93 \mathrm{~dB})$.

improved error resilience. A framework for optimal classification of macroblocks into slice groups and optimal unequal error protection was also proposed. Experimental evaluation showed the superiority of the proposed method in comparison to well-known schemes for transmission of H.264/AVC streams.

\section{ACKNOWLEDGMENT}

This work was partially supported by the European Commission under Contract FP6-511568 3DTV.

\section{REFERENCES}

[1] Information Technology - Coding of Audio-Visual Objects - Part 10: Advanced Video Coding. Final Draft International Standard. ISO/IEC FDIS 14 496-10, 2003.

[2] T. Wiegand, G. J. Sullivan, G. Bjntegaard, and A. Luthra, "Overview of the H.264/AVC video coding standard," IEEE Transaction Circuits and Systems for Video Technology, vol. 13, no. 7, pp. 560-576, 2003.

[3] T. Stockhammer and M. Bystrom, "H.264/AVC data partitioning for mobile video communication," in Proceedings of the International Conference on Image Processing (ICIP '04), pp. 545548, Singapore, October 2004. 
[4] J. Hagenauer, "Rate-compatible punctured convolutional codes (RCPC Codes) and their applications," IEEE Transaction on Communications, vol. 36, no. 4, pp. 389-400, 1989.

[5] O. Harmanci and A. M. Tekalp, "Optimization of h.264 for low delay video communication over lossy channels," in Proceedings of IEEE International Conference on Image Processing (ICIP '04), vol. 5, pp. 3209-3212, Singapore, October 2004.

[6] T. Stockhammer, T. Wiegand, T. Oelbaum, and F. Obermeier, "Video coding and transport layer techniques for H.264/AVCbased transmission over packet-lossy networks," in Proceedings IEEE International Conference on Image Processing (ICIP '03), vol. 3, pp. 481-484, Barcelona, Spain, September 2003.

[7] ITU-T, "Recommentation H.263: video coding for low bit rate communication," 1998.

[8] E. Masala, H. Yang, K. Rose, and J. C. D. Martin, "Ratedistortion optimized slicing, packetization and coding for error resilient video transmission," in Proceedings of DCC Data Compression Conference, pp. 182-191, Snowbird, Utah, USA, March 2004.

[9] S. K. Karande and H. Radha, "Rate-constraint adaptive FEC for video over erasure channels with memory," in Proceedings of IEEE International Conference on Image Processing (ICIP '04), pp. 2539-2542, Singapore, October 2004.

[10] Y. K. Wang, M. M. Hannuksela, and M. Gabbouj, "Error resilient video coding using unequally protected key pictures," in Proceedings of the 8th International Workshop on Very Low Bitrate Video Coding (VLBV'03), pp. 290-297, Madrid, Spain, September 2003.

[11] E. Masala, D. Quaglia, and J. C. D. Martin, "Adaptive picture slicing for distortion-based classification of video packets," in Proceedings of the IEEE Workshop on Multimedia Signal Processing, pp. 111-116, Cannes, France, October 2001.

[12] Q. Qu, Y. Pei, J. W. Modestino, and X. Tian, "Error-resilient wireless transmission using motion-based unequal error protection and intra-frame packet interleaving," in Proceedings of IEEE International Conference on Image Processing (ICIP '04), pp. 837-840, Singapore, October 2004.

[13] S. Wenger and M. Horowitz, "Flexible MB ordering-a new error resilience tool for IP-based video," in Proceedings of International Workshop on Digital Communications (IWDC'02), Capri, Italy, September 2002.

[14] "Joint Model Reference Encoding Methods and Decoding Concealment Methods," JVT-I049d0, San Diego, Calif, USA, September 2003.

[15] S. Wenger, "H.264/AVC over IP.," IEEE Transaction on Circuits and Systems for Video Technology, vol. 13, no. 7, pp. 645-656, 2003.

[16] N. Thomos, N. V. Boulgouris, and M. G. Strintzis, "Wireless image transmission using turbo codes and optimal unequal error protection," IEEE Transaction on Image Processing, vol. 14, no. 11, pp. 1890-1901, 2005.

[17] B. A. Banister, B. Belzer, and T. R. Fischer, "Robust image transmission using JPEG2000 and turbo-codes," IEEE Signal Processing Letters, vol. 9, no. 4, pp. 117-119, 2002.

[18] “The network simulator - ns2," http://www.isi.edu/nsnam/ns/ index.html.

[19] “Jvt reference software version 8.3," http://iphome.hhi.de/ suehring/tml/.

[20] T. Wiegand and B. Girod, Multi-Frame Motion-Compensated Prediction for Video Transmission, Kluwer Academic, San Diego, Calif, USA, 2001, JVT-I049d0.
[21] T. Stockhammer, D. Kontopodis, and T. Wiegand, "Ratedistortion optimization for JVT/H.26l coding in packet loss environment," in Proceedings of Packet Video Workshop, Pittsburgh, Pa, USA, April 2002.

[22] H. Hannu, L. E. Jonsson, R. Hakenberg, et al., "RObust header compression (ROHC): framework and four profiles: RTP, UDP, ESP, and uncompressed," in RFC 3095, Barcelona, Spain, July 2001.

[23] R. Cuny and A. Lakaniemi, "VoIP in 3G networks: an end-toend quality of service analysis," in Proceedings of IEEE Vehicular Technology Conference (VTC '03), pp. 930-934, Jeju, Korea, April 2003.

[24] B. Wang, H. Schwefel, K. Chua, R. Kutka, and C. Schmidt, "On implementation and improvement of robust header compression in UMTS," in Proceedings of the 13th IEEE International Symposium on Personal Indoor and Mobile Radio Communications (PIMRC '02), pp. 1151-1155, Lisboa, Portugal, September 2002.

[25] C. Westphal, "Layered IP header compression architecture for multi-hop compression," in Proceedings of IEEE Global Telecommunications Conference (GLOBECOM '05), St. Louis, Mo, USA, November-December 2005.

[26] R. Sridharan, R. Sridhar, and S. Mishra, "A robust header compression technique for wireless ad hoc networks," ACM SIGMOBILE Mobile Computing and Communication Review, vol. 7, no. 3, pp. 23-24, 2005.

[27] F. Fitzek, S. Rein, P. Seiling, and M. Reisslein, "RObust header compression (ROHC) performance for multimedia transmission over 3G/4G wireless networks," Wireless Personal Communications, vol. 32, no. 1, pp. 23-41, 2005.

Nikolaos Thomos received the Diploma and the Ph.D. degrees from the Electrical and Computer Engineering Department of the Aristotle University of Thessaloniki, Thessaloniki, Greece, in 2000 and 2005, respectively. During his studies, he held teaching and research assistantship positions with the Electrical and Computer Engineering Department of the Aristotle University of Thessaloniki. He was also a Postgraduate Research Fellow with the Informatics and Telematics Institute, Centre for Research and Technology Hellas, Thessaloniki, Greece. Currently, he is a Postdoctoral Research Fellow with the Informatics and Telematics Institute, Centre for Research and Technology Hellas, Thessaloniki, Greece. His research interests include joint source and channel coding, multimedia networking, distributed source coding, space-time coding, wavelets, and digital filters. He is a Member of the IEEE and the Technical Chamber of Greece.

Savvas Argyropoulos received the Diploma degree in electrical and computer engineering from the Aristotle University of Thessaloniki, Thessaloniki, Greece, in 2004, where he is currently pursuing the Ph.D. degree. He is also a graduate Research Assistant with the Informatics and Telematics Institute, Thessaloniki, Greece. His research interests include image and video coding/transmission, multimedia commu-

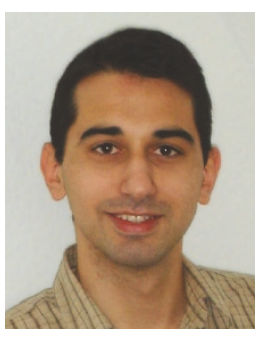
nication, information theory, distributed video coding, and multimodal signal processing. 
Nikolaos V. Boulgouris received the Diploma and the Ph.D. degrees from the Electrical and Computer Engineering Department of the Aristotle University of Thessaloniki, Greece, in 1997 and 2002, respectively. Since December 2004, he has been a Lecturer with the Department of Electronic Engineering, Division of Engineering, at King's College London, United Kingdom. From September 2003 to November

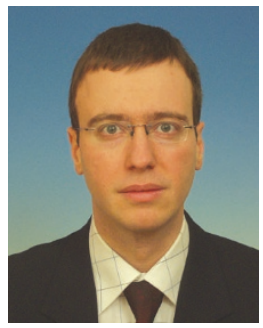
2004, he was a Postdoctoral Fellow with the Department of Electrical and Computer Engineering, University of Toronto, Canada. Previously, he was affiliated to the Informatics and Telematics Institute in Greece. He has participated in several research projects in the areas of pattern recognition, image/video communication, multimedia security, and content-based indexing and retrieval. $\mathrm{He}$ is a Member of the IEEE and the British Machine Vision Association.

Michael G. Strintzis received the Diploma degree in electrical engineering from the National Technical University of Athens, Athens, Greece, in 1967 and the M.A. and Ph.D. degrees in electrical engineering from Princeton University, Princeton, NJ, in 1969 and 1970, respectively. He joined the Electrical Engineering Department, University of Pittsburgh, Pittsburgh, $\mathrm{Pa}$, where he served as an Assistant Professor from 1970

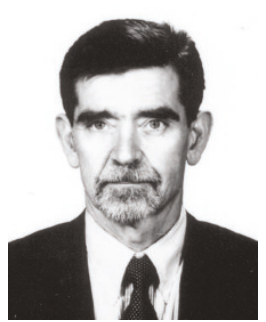
to 1976 and as an Associate Professor from 1976 to 1980. During that time, he worked in the area of stability of multidimensional systems. Since 1980, he has been a Professor of electrical and computer engineering at the Aristotle University of Thessaloniki, Thessaloniki, Greece. He has worked in the areas of multidimensional imaging and video coding. Over the past ten years, he has authored over 100 journal publications and over 200 conference presentations. In 1998, he founded the Informatics and Telematics Institute, currently part of the Centre for Research and Technology Hellas, Thessaloniki.He was awarded the Centennial Medal of the IEEE in 1984 and the Empirikeion Award for Research Excellence in Engineering in 1999. 